\title{
The Application and Influence of Hospice Care Among Patients with Advanced Esophageal Cancer
}

\author{
Zheng-Fang $\mathrm{Li}^{1,2}$ \\ Meng-Jie Liu ${ }^{1,3}$ \\ 'School of Nursing, Southwest Medical \\ University, Luzhou, Sichuan, 646000, \\ People's Republic of China; ${ }^{2}$ Chest \\ Cancer Center, Chongqing University \\ Cancer Hospital, Chongqing, 400030, \\ People's Republic of China; ${ }^{3}$ Department \\ of Nursing, Affiliated Hospital of \\ Southwest Medical University, Luzhou, \\ Sichuan, 646000, People's Republic of \\ China
}

\begin{abstract}
Objective: To investigate the application effect of hospice care in patients with advanced esophageal cancer (EC), provide a practical basis for improving sleep quality, dignity, and subjective well-being, and relieving depression and anxiety in patients with advanced EC.

Methods: A randomized cluster sampling method was used to select 60 patients with advanced EC who received routine intervention (control group) and 64 patients with advanced EC who received hospice care (study group). The intervention time was three months, and the self-rated anxiety scale (SAS) before and after the interventions was compared between the two groups. The Hamilton Depression Scale (HAMD), the Pittsburgh Sleep Quality Index (PSQI), the General Well-being Scale (GWB), the Patient Dignity Scale (PDI), and patients' physical pain state were recorded.

Results: No significant differences were found in the HAMD, SAS, PSQI, GWB, or PDI scores between the two groups before the interventions $(\mathrm{P}>0.05)$; after the interventions, the HAMD, SAS, PSQI, and PDI scores of the two groups were significantly decreased, and the HAMD, SAS, PSQI, and PDI scores of the study group were lower compared with the control group. The GWB scores of the two groups were significantly increased, and those of the study group were significantly higher compared with the control group; the difference was statistically significant $(\mathrm{P}<0.05)$. After the interventions, pain grades of III and above decreased in both groups to grade II, and pain sensation in the study group was lower compared with the control group; the difference was statistically significant $(\mathrm{P}<0.05)$.

Conclusion: Hospice care can reduce the level of pain related to depression and anxiety in patients with advanced EC and improve their sleep quality, as well as their sense of dignity and subjective well-being.
\end{abstract}

Keywords: advanced esophageal cancer, hospice care, sleep quality, anxiety, depression, pain

\section{Introduction}

In China, esophageal cancer (EC) has a high incidence, high mortality, and low survival rate. Patients in the early stage of the disease often show no specific symptoms but as the condition progresses, symptoms or signs, such as dysphagia, sternal pain, and choking of the pharynx arise. Approximately $70-80 \%$ of patients have been diagnosed in the middle and late stages of the disease; ${ }^{1}$ late-stage patients can be changed due to the morphology of lesions. Lymph-node metastasis is a type of malignant tumor that can cause serious harm and produce different coexisting symptoms.

As in the case of most cancers, patients with advanced EC have a high risk of metastasis. In the case of lung metastasis, patients may suffer from coughing,
Correspondence: Meng-Jie Liu School of Nursing, Southwest Medica University, Department of Nursing Affiliated Hospital of Southwest Medical University, Luzhou, Sichuan, 646000,

People's Republic of China

Email liumengjie80@I63.com 
dyspnea, hemoptysis, and chest pain; in the case of bone metastasis, symptoms including systemic bone pain and fractures may occur. Patients with clinically advanced EC often experience an immune stress response, anxiety, and depression due to pathological factors or as a result of the side effects of biological therapy and chemotherapy. Studies note that the incidence of anxiety and depression in EC patients is higher compared with other cancer patients $^{2}$ and poor sleep quality ${ }^{3}$ seriously affects the overall treatment effect and physical and mental health of patients. Therefore, precise and scientific nursing intervention should be adopted for these patients to reduce their physical and mental burdens and to improve their quality of life and physical and mental well-being.

With the continuous progress of clinical nursing concept, routine nursing mode has been difficult to meet the diversified nursing needs of patients with advanced EC. ${ }^{4}$ Hospice care is a type of people-oriented palliative care, the core aspect of which is to provide patients with "body, mind, social, and spiritual" intervention; this is performed by providing pain control to patients with clinical symptoms and psychological distress, ${ }^{5}$ thus ensuring a sense of calm and providing comfort and dignity to those in the final stage of life, a process that has achieved good results in clinical intervention for patients with advanced cancer. ${ }^{6,7}$ However, existing studies have primarily focused on the impact of hospice care on the psychological status and quality of life of patients with advanced EC, ${ }^{8,9}$ while the multi-faceted impact of hospice care on patients with advanced EC has not yet been reported. Accordingly, the current study explored the application effect of hospice care among patients with advanced EC to further enrich the application value of hospice care for patients with malignant tumors while at the same time providing more humane services to patients with EC.

\section{Materials and Methods Demographics}

Selected by a random cluster sampling method from January-September 2019, from the Cancer Hospital Affiliated to Chongqing University, 60 patients with advanced EC who received the conventional intervention treatment were selected as the control group. From January-September 2020, choose hospice care of 64 cases of patients with advanced EC for the team, when patients have a willingness to hospice care, contact with patients and their families, by the researchers in this study, and sign an informed consent form.
The inclusion criteria were as follows: 1) patients who met the diagnostic criteria of advanced $\mathrm{EC}^{10}$ as confirmed by histopathological examination; 2) patients had Tumor Node Metastasis (TNM) stage III and IV; 3) all patients voluntarily participated in and actively cooperated with the study process after being informed of the study's purpose in detail; 4) two specialists jointly diagnosed that the survival time as being less than six months.

The exclusion criteria were as follows: 1) severe water and electrolyte disturbances and the presence of acute infection; 2) complications with serious damage to primary organ functioning; 3 ) a TNM stage $\leq$ II; 4) patients with other primary malignant tumors; 5) patients who could not be fed orally; 6) patients with an estimated survival time of fewer than three months.

\section{Method}

The two groups received intervention treatment in our hospital for three months after diagnosis, and the hospice patients were all in the same area of the ward. The control group received routine intervention treatment including diet control, daily routine guidance, physical symptom monitoring, rehabilitation training, and health education. The patients in this group were instructed to exercise properly to promote general blood circulation. In addition, the mental health status of the patients was carefully monitored and those who experienced negative emotions were provided with guidance and humanistic care as needed.

The study group received hospice care intervention treatment as follows. (1) Basic patient information was collected through direct communication with the patients or their families, friends, and neighbors, by examining their medical examination reports and medical records, and through other means to understand the patient's medical condition and history, psychological mood, character, and behavioral features. (2) The patients' mental state was evaluated based on the basic information collected; patients were classified based on their dependence on people/objects around them and their perception of their own role. Concurrently, behaviors, people, events, and aspects that could directly or indirectly affect patients were identified to deliver any additionally required intervention. (3) In terms of delivering psychological care, the ward was decorated in advance (based on the patient's preferences) in a simple, satisfactory, and affordable manner, and flowers, fruits, and vegetables were provided to liven up the ward, alleviate negative emotions, and encourage patients to actively cooperate in their treatment. (4) In terms of social support, patients were encouraged to communicate with their friends 
and families to mitigate the negative emotions that arose from being alone. In addition, patients' friends and families were reminded to encourage them to become actively involved in their treatment. (5) Relaxation interventions included instructing patients to perform deep breathing and relaxation exercises every morning and evening, during which they would lie down in a comfortable resting state with their legs naturally apart, slowly inhale as much air as possible through their nostrils and pause for 1-2 $\mathrm{s}$ before slowly exhaling as much inhaled air as possible. This helped them to relax both their body and mind. (6) Pain management was delivered by monitoring pain during the intervention process. The attending physician was informed whenever a patient was suffering from pain that required analgesic drugs to stop it from affecting their daily life. The dosage, time, and frequency of the analgesic drugs provided were based on the physician's advice and helped to ensure that patients received daily rest. (7) Targeted nursing was delivered during the intervention by having rehabilitation nursing personnel monitor patients' digestive tract symptoms and providing targeted nursing accordingly. In case of coughing, feebleness, coarse rales, foreign-body obstruction in the esophagus, or hemorrhage, patients received interventions including sputum suction, postural drainage, and establishing hemostasis. In addition, patients were monitored for infections, and regular secretion samples were taken throughout the rehabilitation process for bacterial colony detection. (8) Rehabilitation training was based on the advice of the attending physician, where patients were instructed to perform gentle exercises once their health began improving including active or passive rotation, flexion, extension, and muscle stretching for toes, ankles, hip joints, and knee joints, as well as full-body training, such as bedside sitting down and standing up, and standing and sitting balance. In addition, based on each patient's health condition as it related to dysphagia and hoarseness, they were instructed to perform rehabilitation training of the swallowing function including training that strengthened breathing, lip, and tongue muscles, as well as phonation. The exercises were selected based on each patient's physical condition and were performed for 10-20 min at a time, three times per week. The effect of the three-month intervention was evaluated for both groups.

\section{Outcome Measures}

Patients who could not be assessed independently at the final stage were reviewed using answers provided by their family members.

\section{Mental State}

(1) Anxiety: Before and after the intervention, a self-rating anxiety scale ${ }^{11}$ (SAS) was used to evaluate patients' levels of anxiety. The Cronbach's alpha $(\alpha)$ coefficient was 0.879 , the retest reliability was 0.945 , and the internal consistency coefficient was 0.574 . The scale had a total of 20 items, with a four-grade score and a total score of $20-80$ points. The higher the score, the greater the level of anxiety.

(2) Depression: Depression levels among patients were evaluated using the Hamilton Depression Scale (HAMD). ${ }^{12}$ Cronbach's $\alpha$ coefficient was 0.806 , the retest reliability was 0.921 , and the internal consistency coefficient was 0.566 . There were 24 items included in the scale with a total score of $0-81$ points. The higher the score, the more significant the level of depression.

(3) Sleep quality: The patients' sleep quality was evaluated using the Pittsburgh Sleep Quality Index (PSQI), ${ }^{13}$ which included 18 items. Aggregate scores of $\leq 5,6-10$, $11-15$, and $\geq 16$ suggested good, moderate, poor, and very poor sleep quality, respectively.

\section{Happiness and Self-Esteem}

(1) Happiness: Patients' level of happiness was evaluated before and after the intervention using the General WellBeing Scale (GWB). ${ }^{14}$ The retest reliability was 0.85 , the internal consistency coefficient was 0.93 , and Cronbach's $\alpha$ coefficient was 0.802 , respectively. There were 18 items included in the scale, among which four items had a total score of 4-10 points, and the remaining 14 items had a 6-level score with a total score of 18-94 points. The higher the score, the happier the patient felt.

(2) Self-esteem: Patients' level of self-esteem was evaluated before and after the intervention using the Patient Dignity Inventory (PDI). ${ }^{15}$ Assess client's self-esteem, the overall Cronbach's $\alpha$ coefficient and splitter reliability were 0.924 and 0.885 , respectively. There were 25 items included in the scale, all of which were graded according to six levels, and the total score was $25-150$ points. The higher the score, the lower the self-esteem of the patient.

(3) Perception of pain: Patients' pain perception was evaluated before and after the intervention using the World Health Organization pain rating scale,${ }^{16}$ in which grade I indicated no pain, grade II reflected mild and intermittent pain that required no analgesic medication, grade III was moderate, persistent pain that required intermittent analgesic medication and disrupted normal daily routines, grade IV was severe, persistent pain that required long-term analgesic medication and 
Table I General Information of Patients in the Two Groups

\begin{tabular}{|l|l|c|c|c|}
\hline & & Control Group (N=60) & Study Group (N=64) & P \\
\hline Gender & Male & $\begin{array}{l}38(63.33 \%) \\
22(36.67 \%)\end{array}$ & $\begin{array}{l}40(62.5 \%) \\
24(37.5 \%)\end{array}$ & 0.76 \\
\hline Age & Female & $52.49 \pm 3.58$ & $52.67 \pm 3.72$ & 0.55 \\
\hline Diameter of tumor cm & & $1.25 \pm 0.36$ & $1.31 \pm 0.42$ & 0.79 \\
\hline TNM Stage & Stage III & $24(40 \%)$ & $26(40.6 \%)$ & $38(59.4 \%)$ \\
\hline Zubrod score before intervention & & $36(60 \%)$ & $1.49 \pm 0.21$ & 0.68 \\
\hline Zubrod score 3 months after intervention & & $1.47 \pm 0.25$ & $3.64 \pm 0.13$ & 0.89 \\
\hline
\end{tabular}

severely disrupted normal daily routines, and grade $\mathrm{V}$ pain was more intense than grade IV and threatened the patient's vital signs (eg, blood pressure, pulse, and heart rate).

Physical fitness status: The physical status (PS) status of cancer patients was evaluated using the Zubrod scoring system ${ }^{17}$ in which a score of $0-4$ changed from indicating intact function and no discomfort to being bedridden.

\section{Statistical Methods}

In this study, the statistical analysis and processing of data were conducted using the SPSS Statistics (v.22.0) software. Measurements and enumeration data were subject to $\mathrm{T}$ and chi-squared $\left(\chi^{2}\right)$ tests and were expressed as $\bar{x} \pm s$ and $n(\%)$, respectively. Ranked data were analyzed using the rank-sum test; $\mathrm{P}<0.05$ was considered to indicate statistical significance.

\section{Results}

\section{General Data Comparison Between the Two Groups}

There was no statistical significance in terms of gender, age, tumor diameter, TNM stage, and PS status score before and after the intervention $(\mathrm{P}>0.05)$ as shown in Table 1.

\section{Mental State Comparison Between the Two Groups Before and After the Intervention}

Before the intervention, there were no statistically significant differences in the HAMD, SAS, and PSQI scores between the two groups $(\mathrm{P}>0.05)$. After the intervention, the scores of the HAMD, SAS, and PSQI in the two groups were significantly reduced, and the scores of the HAMD, SAS, and PSQI in the study group were lower than those in the control group, and the differences were statistically significant $(\mathrm{P}<0.05$; see Table 2$)$.

\section{Happiness and Self-Esteem Between the Two Groups Before and After the Intervention}

Before the intervention, there were no statistically significant differences in the GWB and PDI scores between the two groups $(\mathrm{P}>0.05)$. After the intervention, the GWB scores of the two groups were significantly improved, and the GWB scores of the study group were higher than those of the control group; the PDI scores of the two groups were significantly decreased, and the PDI scores of the study group were lower than those of the control group, and the differences were statistically significant $(\mathrm{P}<0.05$; see Table 3$)$.

Table 2 Comparison of HAMD, SAS and PSQI Scores Between the Two Groups Before and After Intervention ( $\overline{\mathrm{x}} \pm \mathrm{s}$, Point)

\begin{tabular}{|l|c|c|c|c|c|c|c|}
\hline \multirow{2}{*}{ Group } & \multirow{2}{*}{} & \multicolumn{2}{|c|}{ HAMD } & \multicolumn{2}{c|}{ SAS } & \multicolumn{2}{c|}{ PSQI } \\
\cline { 3 - 7 } & & $\begin{array}{c}\text { Before the } \\
\text { Interventions }\end{array}$ & $\begin{array}{c}\text { After the } \\
\text { Interventions }\end{array}$ & $\begin{array}{c}\text { Before the } \\
\text { Interventions }\end{array}$ & $\begin{array}{c}\text { After the } \\
\text { Interventions }\end{array}$ & $\begin{array}{c}\text { Before the } \\
\text { Interventions }\end{array}$ & $\begin{array}{c}\text { After the } \\
\text { Interventions }\end{array}$ \\
\hline Study group & 64 & $53.14 \pm 4.39$ & $28.59 \pm 2.61 *$ & $52.16 \pm 4.29$ & $27.53 \pm 2.69 *$ & $17.35 \pm 0.95$ & $8.69 \pm 1.26 *$ \\
Control group & 60 & $52.83 \pm 4.11$ & $35.27 \pm 3.59 *$ & $51.58 \pm 4.15$ & $35.49 \pm 3.18^{*}$ & $17.12 \pm 0.89$ & $13.25 \pm 1.80^{*}$ \\
$\mathrm{t}$ & & 0.382 & 11.162 & 0.721 & 14.173 & 1.310 & 15.392 \\
$P$ & & 0.703 & 0.000 & 0.473 & 0.000 & 0.193 & 0.000 \\
\hline
\end{tabular}

Notes: Compared with the same group before the intervention $* P<0.05$. 
Table 3 Comparison of GWB and PDI Scores Between the Two Groups Before and After Intervention ( $\bar{x} \pm s$, Point)

\begin{tabular}{|l|c|c|c|c|c|}
\hline \multirow{2}{*}{ Group } & \multirow{2}{*}{$\mathbf{n}$} & \multicolumn{2}{|c|}{ GWB } & \multicolumn{2}{c|}{ PDI } \\
\cline { 3 - 6 } & & $\begin{array}{c}\text { Before the } \\
\text { Interventions }\end{array}$ & $\begin{array}{c}\text { After the } \\
\text { Interventions }\end{array}$ & $\begin{array}{c}\text { Before the } \\
\text { Interventions }\end{array}$ & $\begin{array}{c}\text { After the } \\
\text { Interventions }\end{array}$ \\
\hline Study group & 64 & $47.52 \pm 5.67$ & $70.28 \pm 4.19 *$ & $119.43 \pm 6.85$ & $80.76 \pm 4.58^{*}$ \\
Control group & 60 & $47.68 \pm 5.70$ & $61.18 \pm 5.88^{*}$ & $118.59 \pm 6.65$ & $91.43 \pm 6.19 *$ \\
$\mathrm{t}$ & & 0.111 & 7.052 & 0.489 & 10.958 \\
$P$ & & 0.912 & 0.000 & 0.626 & 0.000 \\
\hline
\end{tabular}

Notes: Compared with before intervention in the same group $* P<0.05$.

\section{Pain Ratings Between the Two Groups Before and After the Intervention}

Before the intervention, there was no statistically significant difference in the pain rating between the two groups $(\mathrm{Z}=$ 0.655 and $P>0.05$ ). After the intervention, the grade III and above pain grades in the two groups were significantly reduced to grade II, and the pain sensation in the study group was lower than in the control group; the difference was statistically significant $(Z=2.352$ and $P<0.05$; see Table 4$)$.

\section{Discussion}

Although surgical treatment is an effective approach for the treatment of EC at present, and the survival cycle and quality of life of patients can be significantly improved after surgical treatment, ${ }^{18,19}$ patients with advanced EC are prone to developing depression and anxiety due to the side effects and pathological pain caused by long-term chemotherapy, biotherapy, and radiotherapy. Additionally, negative emotions, such as low self-esteem, can stimulate the body's immune stress response, which can rapidly reduce immune resistance. ${ }^{20-22}$ This can lead to an increase in the pain experienced by the patient and overstrain them, thereby affecting their quality of life and their physical and mental well-being. Scientific and effective nursing intervention is of great significance to the end-of- life experience of patients with advanced EC. ${ }^{23}$ Routine care is a nursing service for patients with a survival time $\geq 6$ months, while hospice care represents nursing and spiritual care services for patients with a survival time $\leq 6$ months that is supplemented by appropriate medical treatment to reduce disease symptoms, delay disease progression, and improve a patient's quality of life. With the continuous improvement of clinical patients' nursing requirements, conventional nursing modes have failed to meet the diverse needs of patients, making it difficult to maximize the intervention effect and, accordingly, affecting prognosis level and patients' quality of life. ${ }^{24}$

As a human-oriented care intervention mode, the ultimate goal of hospice care is to treat terminally ill patients and their families as a basic medical care unit, provide continuous and uninterrupted medical services, assist patients to choose a fulfilling and comfortable life, and relieve their physical and psychological pain as much as possible. Its purpose is based on respecting the natural law of life, maintaining the dignity of patients, implementing whole-person care and appropriate treatment, ensuring physical and mental comfort, and assisting patients to die peacefully. Based on patients' personal basic information, medical history, disease knowledge, treatments and interventions targeted personalized intervention plan after fully

Table 4 Comparison of Pain Grading Between the Two Groups Before and After Intervention [Num., (\%)]

\begin{tabular}{|c|c|c|c|c|c|c|c|}
\hline Time & Group & $\mathbf{n}$ & Level I & Level II & Level III & Level IV & Level V \\
\hline \multirow[t]{4}{*}{ Before the interventions } & Study group & 64 & $0(0.00)$ & $0(0.00)$ & $40(62.50)$ & $22(34.38)$ & $2(3.13)$ \\
\hline & Control group & 60 & $0(0.00)$ & $0(0.00)$ & $40(66.67)$ & $20(33.33)$ & $0(0.00)$ \\
\hline & Z & & & & & & 0.655 \\
\hline & $P$ & & & & & & 0.531 \\
\hline \multirow[t]{4}{*}{ After the interventions } & Study group & 64 & $0(0.00)$ & $24(37.50)^{*}$ & $34(53.13)^{*}$ & $6(9.38)^{*}$ & $0(0.00)^{*}$ \\
\hline & Control group & 60 & $0(0.00)$ & $10(16.67)^{*}$ & $36(60.00)^{*}$ & $14(23.33) *$ & $0(0.00)$ \\
\hline & Z & & & & & & 2.352 \\
\hline & $P$ & & & & & & 0.013 \\
\hline
\end{tabular}

Notes: Compared with the group before intervention $* P<0.05$. 
understand, and thus to the greatest degree avoid dying patients with advanced EC phase all kinds of unexpected events, through comprehensive intervention processes to best improve patient comfort and relieve their anxiety, depression, and possible inferiority complexes, enabling them to retain their dignity through their final stage of life. The results of this study showed that after completing an intervention, the HAMD, SAS, PSQI, and PDI scores of the study group were lower compared with the control group; the study group's GWB score was higher compared with the control group, which corresponded to the results in studies conducted by Yue et $\mathrm{al}^{25}$ and Xie et al. ${ }^{26}$

Based on the above results, hospice care intervention can improve the depression of patients with advanced EC and lessen their anxiety and other negative emotions, promote their physical rehabilitation, and improve both their physical and mental comfort. This may be because people-centered and holistic psychological care can reduce the negative psychological emotions caused by disease and make patients feel more comfortable Additionally, comprehensive intervention related to disease risk factors, psychological factors, and pathological pain is also carried out as part of this treatment approach. All types of nursing processes are interconnected and mutually promoted when delivering hospice care. Furthermore, based on routine care, the anxiety and depression of advanced EC patients were shown to have been improved; patients' pain was also reduced and their sense of dignity and happiness was enhanced.

In conclusion, hospice care intervention for patients with advanced EC can reduce feelings of depression, relieve pain, and improve patients' sense of dignity and happiness. Their anxiety can be diminished and their quality of sleep can also be improved. All of these factors together indicate the broad clinical application prospects of this type of care.

\section{Ethics Approval and Consent to Participate}

This study was conducted in accordance with the declaration of Helsinki. This study was conducted with approval from the Ethics Committee of Affiliated Chongqing University Cancer Hospital. A written informed consent was obtained from all participants.

\section{Consent for Publication}

Consent for publication was obtained from every individual whose data are included in this manuscript.

\section{Funding}

Major Science and Technology Projects of Sichuan Provincial Department of Science and Technology in 2020 (No. 2020YFS0515); Key Research Project of Sichuan Provincial Health Commission (No. 20PJ133); Key Project of Sichuan Provincial Department of Education (No. 18SA0300); 2017 Luzhou Municipal People's Government - Southwest Medical University Science and Technology Strategic Cooperation Project (No. 2017LZXNYD-T08); National Cancer Center Climbing Fund Scientific Research Project (No. NCC201822B730).

\section{Disclosure}

All authors have contributed significantly to the manuscript and declare that the work is original and has not been submitted or published elsewhere. None of the authors have any financial disclosure or conflicts of interest.

\section{References}

1. Wang F, Fang QX. Current status and future prospect of internal medicine treatment for advanced esophageal cancer. Chin J Oncol. 2016;38(09):655-659.

2. Brintzenhofe-Szoc KM, Levin TT, Li Y, et al. Mixed anxiety/depression symptoms in a large cancer cohort: prevalence by cancer type. Psychosomatics. 2009;50:383-391. doi:10.1176/appi.psy.50.4.383

3. Laursen L, Schønau MN, Bergenholtz HM, et al. Table in the corner: a qualitative study of life situation and perspectives of the everyday lives of oesophageal cancer patients in palliative care. BMC Palliat Care. 2019;18(1):60. doi:10.1186/s12904-019-0445-2

4. Heng YJ, Yang HY, Wu Q, et al. Tim-3 expression on T cell surface in patients with esophageal cancer and its clinical significance. Chin $J$ Cancer Biother. 2019;26(03):312-316.

5. Liu MJ. Research on the Construction of the Palliative Care Model for ICU Patients at the End of Life [D]. Third Military Medical University; 2016.

6. Zhang HF. Analysis of hospice care needs and influencing factors in patients with advanced non-small cell lung cancer. Hebei Med J. 2020;42(22):118-121.

7. Greenstein JE, Policzer JS, Shaban ES. Hospice for the primary care physician. Prim Care. 2019;46(3):303-317. doi:10.1016/j. pop.2019.04.002

8. Zhao SP, Zhang J, Yao X. Impact Assessment of palliative care for advanced psychological status and quality of life in patients with esophageal cancer. Chin Oncol Clin Rehabil. 2016;23(08):980-982.

9. Xu BF. Effect of hospice care combined with comfort care on quality of life of patients with advanced esophageal cancer. Contemp Nurse. 2020;27(07):93-95.

10. Lu YY, Lu LG, Qian BY. Guidelines for the treatment of esophageal and gastric cancers. Gastroenterology. 2012;17(03):173-175.

11. Wan QL, Yang YL. Effects of paroxetine on depression and immune function in elderly patients after radical resection of esophageal cancer. Int J Psychiatry. 2019;46(05):138-140.

12. Luo F, Chen YS, Li X, et al. Correlation between the depression and serum leptin, ghrelin levels in patients with esophageal cancer. J Xinxiang Med Univ. 2019;36(003):258-261. 
13. Cao Y, Gao YF, Zhang QY, et al. Effect of cognitive behavioral therapy for insomnia in esophageal cancer patients with insomnia. J Clin Med Pract. 2019;23(10):22-24,28.

14. Chen CH, Yang ZY. Efficacy of positive emotional experience intervention in patients with cervical cancer. Chin J Clin Oncol Rehabil. 2019;26(10):104-106.

15. Wang L, Zhang X, Han J, et al. The relationship between the awareness of disease status and quality of life anxiety depression dignity in Chinese cancer patients. Hebei Med. 2019;25(12):1945-1950.

16. Han SY, Wang YQ, Lu DP. Clinical observation of pain and anxiety in patients with advanced esophageal cancer treated by integrated traditional Chinese and western medicine. World J Integr Tradit Chin West Med. 2020;15(03):131-133+137.

17. West HJ, Jin JO. Performance status in patients with cancer. JAMA Oncol. 2015;1(7):998. doi:10.1001/jamaoncol.2015.3113

18. Ueda Y, Chujo M. Eating behaviors of postoperative esophageal cancer patients during the first year after surgery. Yonago Acta Med. 2020;63(3):173-182. doi:10.33160/yam.2020.08.005

19. Yu YF, Zhu PQ. Effect of pain nursing combined with hospice care on quality of life of patients with advanced gastric cancer. Chin J Cancer Prev Treat. 2019;26(S1):216.
20. Wang YX, Wang XM, Jiao L, et al. Cognition and the influencing factors of advance directives and end-of-life care of patients with advanced tumors. Med Philos. 2019;40(05):30-34.

21. Zhou S, Zhou RN. Effect of hospice care on quality of life and psychological status of patients with terminal cancer. Guizhou Med. 2020;44(03):501-502.

22. Fu HY, Luo Y, Li HJ, et al. Multidimensional dilemma and solutions of hospice care: taking patients with advanced liver cancer as an example. Med Philos. 2020;13(08):79-83.

23. Cao S, Li YH. Effect of hospice nursing mode on negative negative emotion and quality of life in patients with advanced liver cancer. Mod J Integr Tradit Chin West Med. 2019;28(12):1357-1360, 1368.

24. Lu ML, Luo ZQ. Comparative analysis of the level of impaired dignity in young and middle-aged patients with advanced cancer. J Nurses Dev. 2019;16:1499-1502.

25. Yue PR. Effect of hospice care on sleep quality and quality of life in patients with advanced esophageal cancer. Diabetes World. 2018;15 (10): 189 .

26. Xie FT, Cheng LF. Nursing experience of nursing a patient with esophageal cancer in terminal stage with palliative care. Zhanghua Nurs. 2020;27(1):119-128.
International Journal of General Medicine

\section{Publish your work in this journal}

The International Journal of General Medicine is an international, peer-reviewed open-access journal that focuses on general and internal medicine, pathogenesis, epidemiology, diagnosis, monitoring and treatment protocols. The journal is characterized by the rapid reporting of reviews, original research and clinical studies
Dovepress

across all disease areas. The manuscript management system is completely online and includes a very quick and fair peer-review system, which is all easy to use. Visit http://www.dovepress.com/ testimonials.php to read real quotes from published authors. 